\title{
Determination of Menadione Sodium Bisulfite in Pharmaceutical Preparations by Flow-Injection On-line Photochemical Spectrofluorometry
}

\author{
Qiaohong He, Zhixin Wang, Xiaoxia CaO, Hengwu Chen, ${ }^{\dagger}$ and Yinfen Ke \\ Department of Chemistry, Zhejiang University, Xixi Campus, Hangzhou 310028, P. R. China
}

\begin{abstract}
A flow-injection on-line photochemical spectrofluorometry (FI-PF) was developed for the determination of menadione sodium bisulfite (MSB) using acetone and sodium sulfite as sensitizing reagents. An injected sample band carried by a water stream was on-line merged with a mixed $\mathrm{NaOH}, \mathrm{Na}_{2} \mathrm{SO}_{3}$ and acetone solution in a " $\mathrm{T}$ " connector. It was then driven to pass a knotted PTFE photochemical reactor $(0.5 \mathrm{~mm}$ i.d. $\times 200 \mathrm{~cm}, \mathrm{KR})$ that was freely coiled around a $6-\mathrm{W}$ low-pressure mercury lamp. While passing the KR, MSB was derived into an intensively fluorescent compound that was on-line delivered into a flow-through cell and detected therein at an emission wavelength of $459 \mathrm{~nm}$ and an excitation wavelength of $336 \mathrm{~nm}$. Under optimized conditions a detection limit of $0.38 \mu \mathrm{g} \mathrm{l}^{-1}$ was achieved at a sampling rate of 90 $\mathrm{h}^{-1}$. Eleven determinations of $0.5 \mathrm{mg} \mathrm{l}^{-1}$ and $0.05 \mathrm{mg} \mathrm{l}^{-1} \mathrm{MSB}$ standard solution gave RSDs of $0.75 \%$ and $1.3 \%$, respectively. The calibration curve was linear in the MSB concentration range $0.005-1.5 \mathrm{mg} \mathrm{l}^{-1}$. The proposed method was successfully applied to assay the MSB content in MSB injection.
\end{abstract}

(Received April 27, 2001; Accepted August 3, 2001)

\section{Introduction}

Vitamin $\mathrm{K}$ is a generic term for a group of fat-soluble vitamins consisting of 2-methyl-1,4-naphthoquinone. Vitamin K3 (menadione) is a synthetic vitamin $\mathrm{K}$ and is highly unstable. Menadione is not used for human beings and animals because it has a corrosive effect on the respiratory tract and skin. Menadione sodium bisulfite (MSB) is a water-soluble derivative of menadione. It can be used to cure various diseases, such as obstractive, gallbladderatrophy and newborn bleeding as a required cofactor in the synthesis of zymoplasm in liver. It has also received great attention concerning its anticancer activity. A number of analytical methods have been reported for its determination. Spectrofluorometry is among the frequently used ones. ${ }^{1-6}$ Although MSB is a non-fluorescent compound, it can be converted into a strongly fluorescent compound based on reduction of its hydrolyzed compound, menadione. The reduction of menadione may be conducted either chemically ${ }^{7,8}$ or electrochemically ${ }^{9}$ as well as photochemically. ${ }^{10}$ Torro et $a l .{ }^{11}$ developed a flow injection (FI) spectrofluorometry for the determination of MSB based on chemical reduction of the menadione by a solid-phase reactor packed with metal zinc powder. Despite the fact that a quite high sample throughput $\left(70 \mathrm{~h}^{-1}\right)$ was achieved, the zinc column had to be renewed after a period of application and its manifold was more complex. Photochemical reduction provides more advantage. Recently, Guo et al. ${ }^{12}$ reported an in-situ photochemical spectrofluorometric method for the determination of MSB. In

† To whom correspondence should be addressed.

E-mail: hwchen@mail.hz.zj.cn

X. C. present address: School of Engineering, Zhejiang University of Technology. their study, acetone in cooperation with sodium sulfite was found to be effective to accelerate the photochemical reaction and to increase the sensitivity of photochemically induced fluorescence (PCF) for the determination of MSB. Thus, a detection limit of $0.76 \mu \mathrm{g} \mathrm{l}^{-1}$ was achieved. However, the insitu photochemical reaction required the sample zone to be stopped in the flow-through cell, where it was exposed to monochromatic radiation $(280 \mathrm{~nm})$ from the excitation monochromator. After $120 \mathrm{~s}$, the excitation monochromator was switched to $340 \mathrm{~nm}$ for measuring the PCF intensity. As a result, the sample throughput was less than $30 \mathrm{~h}^{-1}$, and excessive wearing of the mechanical system of the excitation monochromator was caused due to frequent switching of the monochromator back-and-forth during the measurement.

In the present work, the behavior of the flow-injection on-line photochemical-induced fluorescence of MSB was investigated. Using sodium sulfite and acetone as sensitizing reagents, we developed a flow-injection on-line photochemical spectrofluorometry (FI-PCF) approach for the determination of MSB. This approach features high sensitivity, quick sample throughput, simple operation and good reproducibility, and has been successfully applied to assay the MSB content in pharmaceutical preparations.

\section{Experimental}

\section{Apparatus}

Fluorescence measurements were made with a Model RF-540 spectrofluorometer (Shimadzu, Kyoto, Japan) equipped with a xenon lamp. A Model LZ-1010 peristaltic pump (Zhaofa Institue for Lab Automation, Shenyang, China), a Rheodyne 5020 six-part PTFE injector with a $250 \mu$ l sampling loop (Cotati, CA), and a $120 \mu \mathrm{l}$ rectangular quartz flow-through cell 


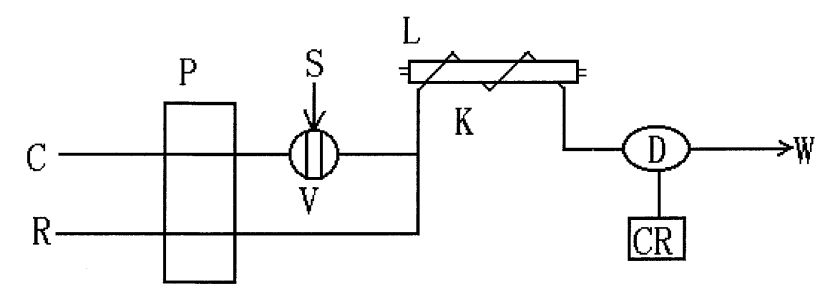

Fig. 1 Experiment setup of FI on-line photochemical spectrofluorometry for the determination of MSB. C, carrier $\left(\mathrm{H}_{2} \mathrm{O}\right)$; $\mathrm{CR}$, chart recorder; D, flow-through cell of spectrofluorometer; K, knotted PTFE photochemical reactor; L, 6-W low-pressure mercury lamp; P, peristaltic pump; R, mixed reagent solution; S, sample solution; $\mathrm{V}$, injection valve; $\mathrm{W}$, waste.

(Shimadzu) were used to set up the FI manifold, as illustrated in Fig. 1. A chart recorder (Dahua Instrument Co., Shanghai, China) was used to record the FI peak traces. The photochemical reactor was home-made by knotting a $0.5 \mathrm{~mm}$ i.d. $\times 200 \mathrm{~cm}$ PTFE tubing, as described in Ref. 13. The knotted reactor was freely coiled around a 6-W low-pressure mercury lamp. PTFE tubing of $0.5 \mathrm{~mm}$ i.d. was used for all connections, and PVC pump tubing with appropriate diameter was employed to propel the solutions.

\section{Reagent}

Menadione sodium bisulfite (MSB) was obtained from Shanghai Reagent Co. (Shanghai, China). A stock solution of MSB (500 $\mathrm{mg} \mathrm{l}^{-1}$ ) was prepared by dissolving the reagent in distilled water and stored in the dark at $4^{\circ} \mathrm{C}$. Before using, the standard solution was calibrated against menadione. ${ }^{14}$ A mixed $\mathrm{NaOH}-\mathrm{Na}_{2} \mathrm{SO}_{3}$-acetone solution was daily prepared by dissolving $4 \mathrm{~g}$ of $\mathrm{Na}_{2} \mathrm{SO}_{3}$ in $100 \mathrm{ml}$ of a $0.1 \%$ (w/v) $\mathrm{NaOH}$ solution containing $0.05 \%(\mathrm{v} / \mathrm{v})$ acetone. All of the used chemicals with the exception of MSB were of analytical grade, and dually distilled water was used throughout.

\section{Procedure}

The excitation and emission wavelengths of the spectrofluorometer were set at 336 and $459 \mathrm{~nm}$, respectively. Before a measurement, the mercury lamp was turned on and the water carrier and the mixed reagent solution were pumped through the photochemical reactor and the flow-through cell and finally to waste drain until a steady baseline was recorded. After sampling for $15 \mathrm{~s}$, the injection valve was switched to the injection position. After $25 \mathrm{~s}$, the valve was switched back to the sampling position for the next cycle. Three replicates were measured for each test solution, and the average peak height was used for quantification.

\section{Results and Discussion}

\section{Preliminary tests}

In preliminary tests, MSB solutions were continuously pumped so as to be mixed on-line with a mixed-reagent solution (both streams had the same flow rate of $2.4 \mathrm{ml} \mathrm{min}^{-1}$ ), and were then subjected to photochemical reactions in a $0.5 \mathrm{~mm}$ i.d. $\times$ $200 \mathrm{~cm}$ PTFE reactor.

Various reagents were examined concerning their capability to sensitize the PCF. Tests showed that although $\mathrm{Na}_{2} \mathrm{SO}_{3}$ could greatly enhance the PCF signals, organic solvents such as methanol, ethanol, acetonitrile, formaldehyde and acetone neither enhanced nor depressed the PCF signals if $\mathrm{Na}_{2} \mathrm{SO}_{3}$ was
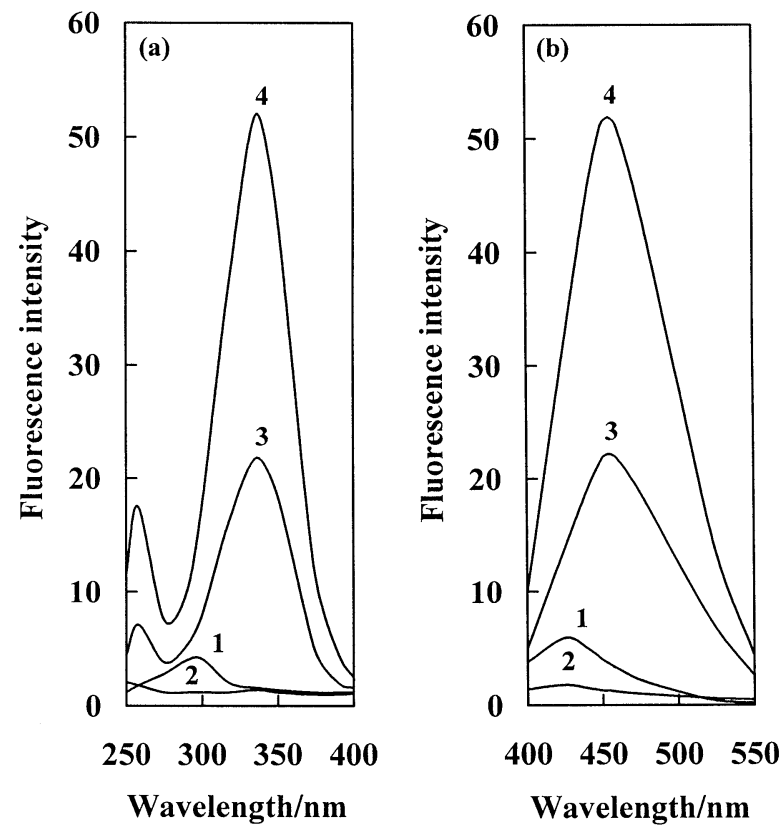

Fig. 2 Fluorescence excitation (a) and emission (b) spectra for the photochemical product of MSB. 1, 4\% $\mathrm{Na}_{2} \mathrm{SO}_{3}-0.1 \% \mathrm{NaOH}-0.05 \%$ acetone blank; 2, $0.5 \mathrm{mg} \mathrm{l}^{-1} \mathrm{MSB}$ in $0.1 \% \mathrm{NaOH} ; 3,0.5 \mathrm{mg} \mathrm{l}^{-1} \mathrm{MSB}$ in $4 \% \mathrm{Na}_{2} \mathrm{SO}_{3}-0.1 \% \mathrm{NaOH}$ mixed reagent; $4,0.5 \mathrm{mg} \mathrm{l}^{-1} \mathrm{MSB}$ in $4 \%$ $\mathrm{Na}_{2} \mathrm{SO}_{3}-0.1 \% \mathrm{NaOH}-0.05 \%$ acetone mixed reagent.

absent. Nevertheless, when $\mathrm{Na}_{2} \mathrm{SO}_{3}$ was present, acetone could remarkably enhance the PCF signals. A variety of surfactants such as sodium lauryl sulfate, sodium dodecyl benzene sulfonate, cetylpyridinium chloride, TritonX-100 and emulsifier Op were also tested. Only non-ionic surfactants, TritonX-100 and emulsifier OP, slightly increased the PCF signals. Thus, acetone in combination with sodium sulfite was selected as sensitizing reagents for FI-PF. Figure 2 shows the excitation and emission spectra of the PCF product of MSB in the presence and absence of sodium sulfite and acetone.

In a previous study on acetone sensitizing photochemical reaction of MSB, ${ }^{12}$ acetone was spiked in the sample solution. This operation is both labor and time consuming. A preliminary test showed that identical PCF signals were observed regardless of whether acetone was spiked into the sample solution or was added into the mixed-reagent solution. Therefore, it was used as one ingredient of the mixed-reagent solution, as described in the Experimental section.

Two types of low-pressure mercury lamps, one without fluorescent powder coating in the inner wall of the lamp tube (maximum emission wavelength was $254 \mathrm{~nm}$ ) and the other with a fluorescent powder coating (maximum emission wavelength was $365 \mathrm{~nm}$ ), were compared as the light resource for photochemical reactions. Although tests showed that both lamps induced a significant PCF signal, the former was a more effective source for the photochemical reaction of MSB. It was thus chosen as the source for PCF.

\section{Optimization of chemical parameters}

In the following study, the FI manifold shown in Fig. 1 was used.

The effect of the $\mathrm{NaOH}$ concentration in the reagent stream on the FI-PF signals was first investigated. Figure 3 shows that regardless of the presence of acetone or not the PCF signals reached the maximum value at an $\mathrm{NaOH}$ concentration of $0.1 \%$. 


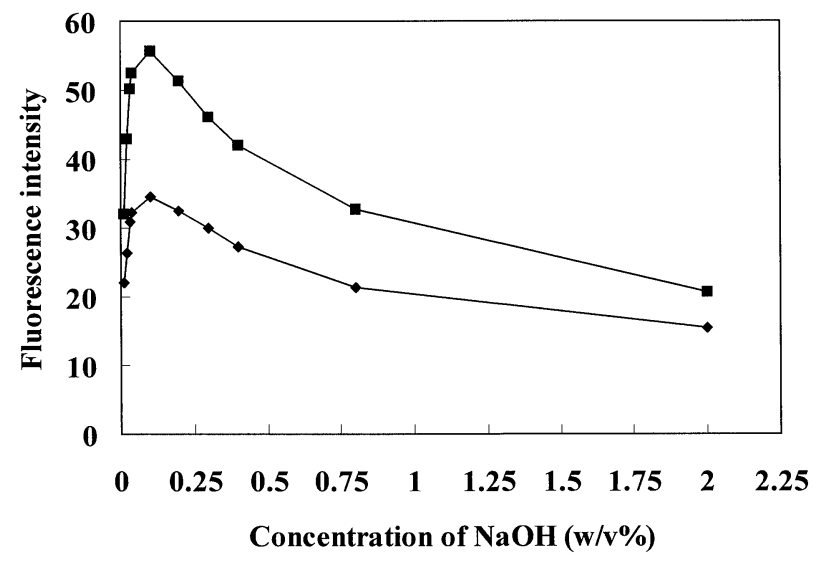

Fig. 3 Effect of the $\mathrm{NaOH}$ concentration in the mixed-reagent solution on the FI-PF signals. The mixed reagent solutions containing no acetone $(\bullet)$ and $0.05 \%$ acetone $(\boldsymbol{\square})$ were on-line merged with an injected sample zone. The concentration of $\mathrm{Na}_{2} \mathrm{SO}_{3}$ in the mixed reagent was $1 \%$. Other experimental conditions were the same as described in Table 1 with the exception of the composition of the mixed reagent solution.

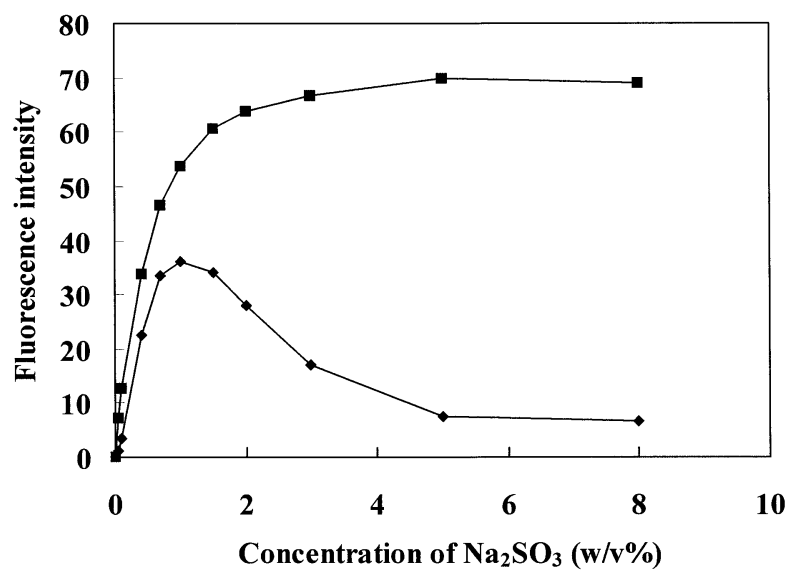

Fig. 4 Effect of the $\mathrm{Na}_{2} \mathrm{SO}_{3}$ concentration in the mixed-reagent solution on the FI-PF signals. The mixed-reagent solutions containing no acetone $(\bullet)$ and $0.05 \%$ acetone $(\boldsymbol{\square})$ were on-line merged with the injected sample zone. The concentration of $\mathrm{NaOH}$ in the mixed reagent was $0.1 \%$. Other experimental conditions were the same as described in Table 1 with the exception of the composition of the mixed-reagent solution.

The influence of the sodium sulfite concentration in a mixedreagent solution on the FI-PF signals was investigated in both the absence and presence of acetone. As shown in Fig. 4, if acetone was not involved in the reaction, the PCF signal was increased along with an increase in the sodium sulfite concentration up to about $1 \%$. After that, a further increase in the sodium sulfite concentration led to a decrease of the FI-PCF signals. However, if $0.05 \%$ acetone was added to the mixed reagent, the situation was absolutely different. The FI-PF signals were quickly increased along with an increase in the $\mathrm{Na}_{2} \mathrm{SO}_{3}$ concentration up to $2 \%$, and then gradually leveled off. The optimum FI-PCF signals were obtained in $\mathrm{Na}_{2} \mathrm{SO}_{3}$ concentrations higher than $3 \%$, which was significantly higher than the optimum $\mathrm{Na}_{2} \mathrm{SO}_{3}$ concentration $(1 \%)$ obtained in the absence of acetone. The interacted influences of acetone and sodium sulfite on the FI-PCF of MSB confirms that the PCF

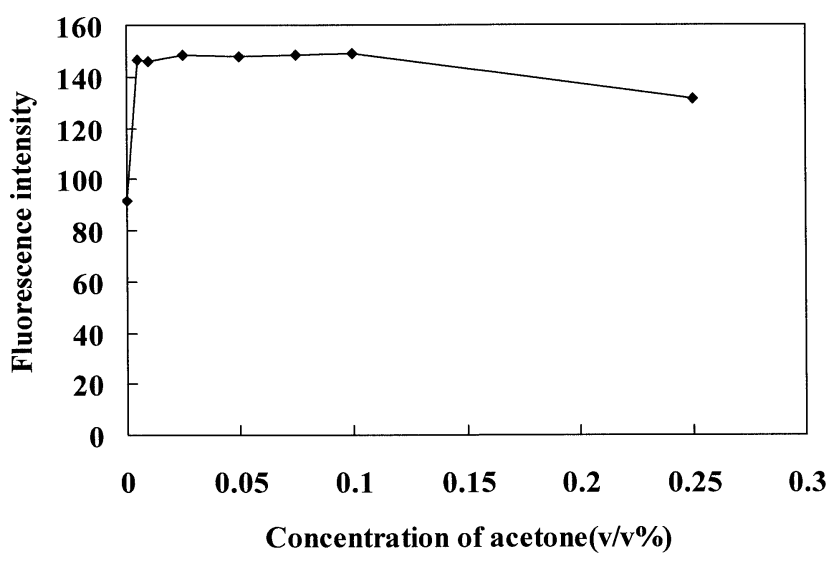

Fig. 5 Effect of the acetone concentration in the mixed-reagent solution on the FI-PF signals. The concentrations of $\mathrm{NaOH}$ and $\mathrm{Na}_{2} \mathrm{SO}_{3}$ in mixed reagent solution were $0.1 \%$ and $4 \%$, respectively. Other experimental conditions were the same as described in Table 1 with the exception of the composition of the mixed-reagent solution.

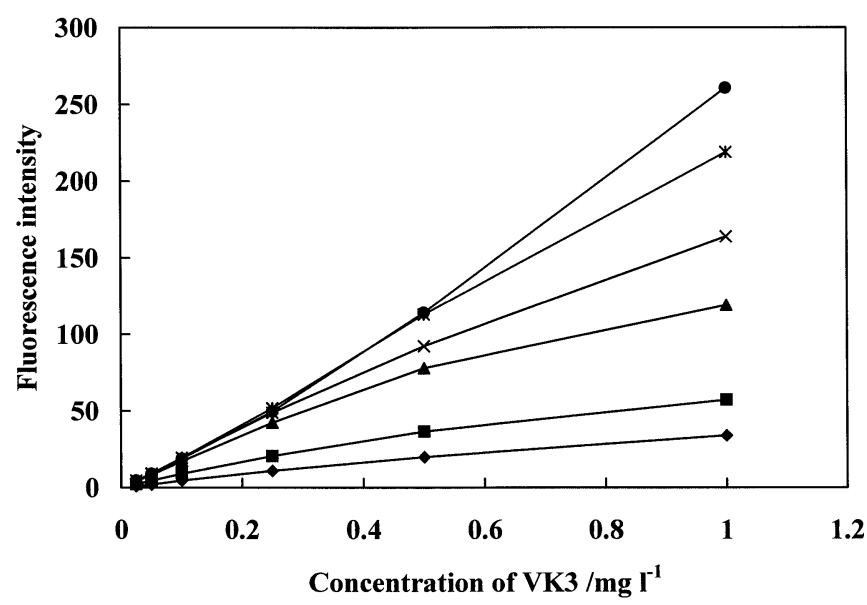

Fig. 6 Calibration curves for MSB obtained by using various knotted photochemical reactors with different PTFE tubing lengths. The tubing length was $25 \mathrm{~cm}(\diamond) ; 50 \mathrm{~cm}(\boldsymbol{\square}) ; 100 \mathrm{~cm}(\boldsymbol{\Delta}) ; 150 \mathrm{~cm}$ $(\times) ; 200 \mathrm{~cm}(*)$, and $300 \mathrm{~cm}(\bullet)$. Other experimental conditions were the same as described in Table 1 with the exception of the reactor tubing length.

mechanism of the drug in the presence of acetone is different from that in its absence, as discussed by Guo et al. in Ref. 12 .

Finally, the effect of the acetone concentration on the FI-PCF was examined. As shown in Fig. 5, the greatest signal was obtained in the acetone concentration range of $0.001-0.1 \%$. Thus, a mixed-reagent solution of $0.1 \% \mathrm{NaOH}, 4 \% \mathrm{Na}_{2} \mathrm{SO}_{3}$ and $0.05 \%$ acetone was prepared for daily use.

\section{Optimization of physical parameters}

In our previous study on the acetone sensitized FI-PCF of reserpine ${ }^{15}$ both the sensitivity and the dynamic linearity of the calibration curve were dependent on the irradiation time at a given acetone concentration. Thus, by using several photochemical reactors with varied tubing length, a set of calibration curves was constructed, at a fixed flow rate, with a series of standard solutions. As shown in Fig. 6, a photochemical reactor with a tubing length of $200 \mathrm{~cm}$ gave the best compromise between sensitivity and linearity. 


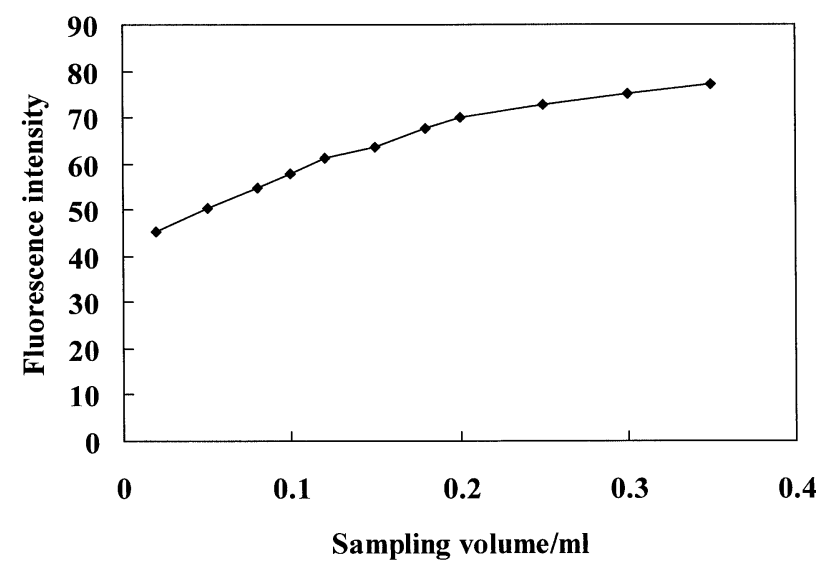

Fig. 7 Effect of the injected sample volume on the FI-PF signals. The experimental conditions were the same as described in Table 1 with the exception of the sampling volume.

The effect of the sampling volume was also tested. The results are shown in Fig. 7. Compromising between the sensitivity and the sampling rate, an injection volume of $250 \mu \mathrm{l}$ was selected.

\section{Analytical performances}

Under the optimized experimental conditions, the analytical performances of the FI-PF method are listed in Table 1. Compared to the in-situ PCF method $^{12}$ and FI spectrofluorometry using a solid-phase reactor, ${ }^{11}$ the present method features a more excellent reproducibility, a higher sample throughput rate, a lower detection limit and a wider linear range. Another advantage of the developed FI-PCF method over the in-situ PCF method lies in that no frequently shift of the excitation wavelength is required.

\section{Application}

The validity of the proposed method was first checked by spike-recovery test. Sample solutions containing $0.20 \mathrm{mg} \mathrm{l}^{-1}$ MSB, prepared with MSB injection, were spiked with a $0.20 \mathrm{mg}$ $1^{-1}$ MSB standard solution. The obtained recovery of the spiked MSB was $102 \pm 1 \%$ (mean \pm S.D., $n=3$ ). The analytical results for a brand of MSB injection obtained by both the proposed method and the pharmacopoeia-specified method ${ }^{14}$ are listed in Table 2. Good agreement in the results was obtained between the two methods.

\section{Conclusion}

It has been demonstrated that the on-line photochemically induced fluorescence of MSB can be greatly enhanced by sodium sulfite and acetone. The proposed flow-injection photochemical spectrofluorometric approach has the merits of a high sample-throughput rate, simple operation, low detection limits, wide linear range and excellent precision. It can be used to determine the MSB content in pharmaceutical preparations.

\section{Acknowledgements}

The Education Department of Zhejiang Provincial Governments is thanked for funding this work.
Table 1 Analytical performance of the FI on-line photochemical spectrofluorometry for MSB the optimized condition $^{\mathrm{a}}$

$\begin{array}{ll}\text { Detection limit }\left(\mu \mathrm{g} \mathrm{l}^{-1}, 3 \sigma\right) & 0.36 \\ \text { Linear dynamic range }\left(\mathrm{mg} \mathrm{l}^{-1}\right) & 0.005-1.5 \\ \text { Regression equation } & F=-2.01+183.1 \times C \\ \text { Correlation coefficient } & 0.9991 \\ \text { Relative standard deviation }(\%, n=11) & 0.75\left(0.5 \mathrm{mg} \mathrm{l}^{-1} \mathrm{MSB}\right) \\ & 1.3\left(0.05 \mathrm{mg} \mathrm{l}^{-1} \mathrm{MSB}\right) \\ \text { Sample throughput rate }\left(\mathrm{h}^{-1}\right) & 90\end{array}$

a. Carrier flow rate, $2.4 \mathrm{ml} \mathrm{min}^{-1}$; the flow rate for the mixed-reagent solution containing $0.1 \% \mathrm{NaOH}, 4 \% \mathrm{Na}_{2} \mathrm{SO}_{3}$ and $0.05 \%$ acetone, 2.4 $\mathrm{ml} \mathrm{min}{ }^{-1}$; photochemical reactor, $0.5 \mathrm{~mm}$ i.d. $\times 200 \mathrm{~cm}$ knotted PTFE tubing freely coiled around a $6-\mathrm{W}$ low-pressure mercury lamp; sampling volume, $250 \mu \mathrm{l}$.

b. $F$, fluorescence intensity at arbitrary unit; $C$, MSB concentration in $\mathrm{mg} 1^{-1}$.

Table 2 Analytical results $\left(\mathrm{mg}^{-1}\right)$ for the determination of MSB in injection

\begin{tabular}{lccc}
\hline \multirow{2}{*}{ Sample } & Labeled & \multicolumn{2}{c}{ Found (mean $\pm \mathrm{SD}, n=3)$} \\
\cline { 3 - 4 } & & Present method & Standard method $^{14}$ \\
\hline $\begin{array}{l}\text { MSB } \\
\text { injection }\end{array}$ & 4 & $4.04 \pm 0.03$ & $4.15 \pm 0.03$ \\
\hline
\end{tabular}

\section{References}

1. T. Perez Rutz, C. Martinez Lozano, V. Tomas, and J. Martin, Analyst, 1999, 124, 197.

2. M. L. Iskander, H. A. A. Medien, and L. H. Khalil, Anal. Lett., 1995, 28, 1513.

3. Z. M. Liu, J. Li, T. Y. You, X. R. Yang, and E. K. Wang, Electroanalysis, 1999, 11, 53.

4. L. Z. Wang, C. S. Ma, X. L. Zhang, and Y. Xu, Microchem. J., 1994, 50, 101.

5. H. X. Ouyang and M. B. Hu, Fenxi Huaxue, 1998, 26, 1158.

6. S. M. Billedeau, J. Chromatogr., 1989, 472, 371.

7. J. J. Berzas Vevado, J. A. Murillo Pulgarin, and M. A. Gomez Laguna, Analyst, 1998, 123, 287.

8. S. M. Billedeau, J. Chromatogr., 1989, 472, 371 .

9. K. Hirauchi, S. Notsumoto, T. Nagaoka, K. Fujimoto, and Y. Suzuki, Bitamin, 1991, 65, 117.

10. J. R. Poulsen and J. W. Birks, Anal. Chem., 1989, 61, 2267.

11. I. Gil Torro, J. V. Garcis Mateo, and J. Martinez Calatayud, Analyst, 1997, 122, 139

12. X. Q. Guo, Y. B. Zhao, and J. G. Xu, Anal. Chim. Acta, 1997, 343, 109.

13. H. W. Chen, J. P. Zhu, X. X. Cao, and Q. J. Fang, Analyst, 1998, 123, 1017.

14. China Pharmacopeia, 1995, ed. China Pharmacopoeial Committee, Renming Weisheng Press House, Beijing, 208.

15. H. W. Chen and Q. H. He, Talanta, 2000, 53, 463. 